\title{
Numerical investigation of dynamic stall phenomenon on a plunging airfoil
}

\author{
F. Ajalli ${ }^{1} \&$ M. Mani ${ }^{2}$ \\ ${ }^{1}$ Department of Aerospace Research Institute, \\ Amirkabir University of Technology, Iran \\ ${ }^{2}$ Department of Aerospace Engineering and \\ Center of Excellence in Computational Aerospace Engineering, \\ Amirkabir University of Technology, Iran
}

\begin{abstract}
The unsteady separated turbulent flow around an oscillating airfoil plunging in a sinusoidal pattern in the regime of low Reynolds number is investigated numerically, employing the URANS approach with advanced turbulence model, $\mathrm{k}-\omega S S T$ transitional. A comparison with experimental data shows that the Transition SST model is capable of predicting the flow characteristics for the increasing cycle while the main difficulty lies in the accurate modeling of the complicated separated flows during the decreasing stroke. The flow development of the dynamic stall is also discussed.
\end{abstract}

Keywords: dynamic stall, plunging airfoil, $k-\omega-S S T$ transitional model.

\section{Introduction}

Dynamic stall has been widely known to significantly affect the performance of a large variety of fluid machinery, such as helicopters, highly maneuverable fighters, gas turbines, and wind turbines. It is well recognized that the dynamic stall process can be categorized into four key stages, i.e. attached flow at low angles of attack, development of the leading edge vortex (LEV), the shedding of the LEV from the suction surface of the blade and the reattachment of the flow [1]. Numerous experimental and computational investigations [2-5] have shown that the unsteady flow can be separating or reattaching over a large portion of the upper surface of the oscillating airfoil and that the predominant feature of the dynamic stall is the formation and rapid convection over the upper surface of the 
airfoil of an energetic leading-edge vortex (LEV), also referred to as the dynamic-stall vortex, which induces a nonlinearly fluctuating pressure field and produces large transient variations in forces and moments that are fundamentally different from their steady-state counterparts.

Although the basic image of the phenomenon has been generally clarified, the physics of this strongly non linear unsteady flow phenomenon has not yet been completely understood and more efforts are needed to advance the knowledge to the level on which we could accurately predict and precisely control the dynamic stall [6]. Most of the previous researches have investigated flows at high Reynolds number $\left(\operatorname{Re} \times 10^{6}\right)$ or high Mach number $(\mathrm{Ma} \geq 0.3)$ which fall into the compressible flow regime. However, dynamic stall at low Reynolds number has distinct features compared with those at high Reynolds number, such as flow transition process, laminar separation and reattachment, etc.

In the recent decades, due to the increased awareness of the environmental issues associated with the fossil fuel based power generation industry, wind industry is drawing more and more attention. Dynamic stall has been a critical phenomenon which has an important effect on the operation of both Horizontal Axis Wind Turbines (HAWT) and Vertical Axis Wind Turbines (VAWT).

The objective of the present study was to investigate the ability of the Unsteady Reynolds-Averaged Navier-Stokes (URANS) method with advanced turbulence model (k- $\omega$ SST transition model) in capturing the dynamic stall at low Reynolds number flows (Reynolds number based on the chord length of the airfoil $R e_{c}$ is of the order of $10^{5}$ ), and to provide a detailed two-dimensional analysis to gain a better understanding of the flow phenomenon. In order to validate the numerical results the experimental measurements of surface pressure distributions was compared with numerical ones at the same condition.

\section{Numerical simulations}

\subsection{Case studied}

The aerofoil employed in the numerical calculations is an E361 airfoil with a chord length of $\mathrm{c}=0.15 \mathrm{~m}$ and maximum thickness of $12 \% \mathrm{c}$ which in this case executes the sinusoidal plunging motion $\mathrm{h}=8(\mathrm{~cm})+\sin (\omega \mathrm{t})$ with reduced frequency $\mathrm{k}=\omega \mathrm{c} / 2 \mathrm{U}_{\infty}=0.14$. The free stream velocity is $\mathrm{U}_{\infty}=10 \mathrm{~m} / \mathrm{s}$ with a turbulence intensity of $0.2 \%$ which corresponds to a chord Reynolds of $\operatorname{Re}_{\mathrm{c}}=1 \times 10^{5}$. The mean angle of oscillations was set up $12 \mathrm{deg}$ with in static stall angle. Numerical set up are based on the experimental tests in order to compare numerical results with experimental data. A more comprehensive description of the experimental setup is detailed in [7].

\subsection{Numerical techniques}

Firstly for the static flow field investigations the RANS approach with advanced turbulent model, namely the k- $\omega$-SST transitional model were used, furthermore

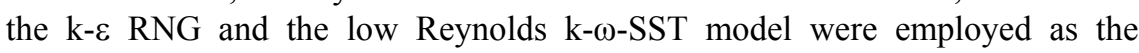


baseline turbulence models to predict the performance of low $\operatorname{Re}$ and fully turbulent approach without transition secondly for the dynamic simulations $\mathrm{k}-\omega$ SST transitional model were applied to the all cases. The transition SST Model is a four- equation turbulence model which is based on the coupling of the SST k- $\omega$ transport equations with two other transport equations, one for the intermittency and the other for the transition onset criteria, in terms of the momentumthickness Reynolds number. This is a reasonably new model and it is expected to predict flows with massive separations more accurately. The FLUENT code used to solve the Reynolds averaged Navier-Stokes equations using finite volume discretization. Second order upwind discretization in space is used, and the resulting system of equations is then solved using the SIMPLE coupled solution procedure until convergence criteria are satisfied. The convergence rate is monitored during the iteration process by means of the residuals of the dependent variables of the governing differential equations. A convergence criteria of $\mathrm{O}(5)$ reduction in all dependent variable residuals is accepted as adequate for the present aerofoil study. Convergence is also checked using the relative differences between two successive iterations for each of the integrated force and moment coefficients. Free stream boundary conditions are used in the upstream, downstream and outer boundaries. No-slip boundary conditions are used at solid surfaces.

\subsection{Grid design}

A hybrid C-grid in figure 1 is used for the calculations and about 256 grid nodes are placed along the aerofoil and they are clustered close to the leading and trailing edges. The height of the first row of cells of the cells bounding the aerofoil is set to be $10^{-5} \mathrm{c}$ which ensures $\mathrm{y}^{+} \leq 1.0$ for the cells immediately adjacent to the aerofoil so that the boundary layer flow can be properly resolved without using a wall function. The height of the cells expands with a growth factor of 1.2 towards the external boundary which is set to be at $25 \mathrm{c}$ from the aerofoil to eliminate the boundary reflections. The whole mesh sums up to be

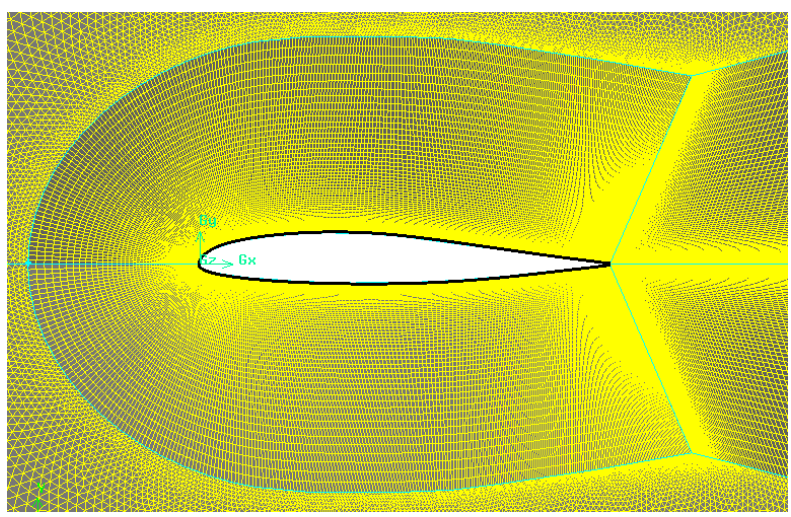

Figure 1: Hybrid grid quality close to the airfoil. 
200,000. In order to simulate the sinusoidal plunging motion of the airfoil, the whole grid oscillates like a rigid body with the same sinusoidal mode as the airfoil. This is achieved by using the dynamic mesh technique [8] with a User Defined Function $(U D F)$ subroutine developed and attached to the Fluent solver to control the movement of the grid points. The numerical time step size is set to be 0.001 . Normally after three oscillation cycles, a perfect periodical solution can be obtained in this study.

\section{Results and discussion}

\subsection{Static airfoil}

In this part of the paper static flow field with increasing incidence at $\mathrm{Re}=10^{5}$ is presented. The identification of static flow field, laminar separation bubble and trailing edge separation serves as a frame of reference for dynamic stall results. Figure 2 shows the computed static pressure coefficient obtained by using different turbulence modeling approaches, compared with the experimentally measured results [9]. There was good agreement between the experiment and k$\omega$ SST transitional model at different angles of attack. According to the experimental data laminar separation bubble (LSB) was formed near the leading edge of the upper surface of the aerofoil, the existence of the separation bubble produced an approximate constant pressure plateau starting from the separation point to the transition region, followed by a sharp pressure rise in the turbulent bubble region blending in to the turbulent boundary layer. As shown in figure 2, k- $\omega$ SST transitional model predicted LSB properly but k- $\varepsilon$ RNG and k- $\omega$ SST turbulence models missed the separation bubble; however there is not significant distinction between lower surface pressure coefficients of the different models. According to the experimental data the stall angle was recorded about 11 degrees whereas k- $\varepsilon$ RNG and k- $\omega$ SST turbulence models over-predicted the stall angle while k- $\omega$ SST transition model under-predicted it. Lastly the stalled pressure coefficient of the airfoil are illustrated in figure $4 \mathrm{c}$, accordingly, the pressure variations of the experimental data and k- $\omega$ SST transitional model included plateau as a result of stall phenomenon, however the computation data of $\mathrm{k}$ $\varepsilon$ RNG and k- $\omega$ SST demonstrated high suction pressure peak near the leading edge that indicated no stall characteristic of the aerofoil.

Information relating to the k- $\omega$ SST solution at $\alpha=4$ deg are provided in figure $3 \mathrm{a}$, according to the results of this model, at 4deg angle of attack, laminar separation occurred on the upper surface at about $60 \%$ chord position from the leading edge. Because the adverse pressure gradient was modest, the separated flow was able to reattach to the surface. As the angle of attack increased, figure $3 \mathrm{~b}$, the adverse pressure gradient aft of the point of suction peak became stronger and the separation point moved toward the leading edge. The stronger pressure 


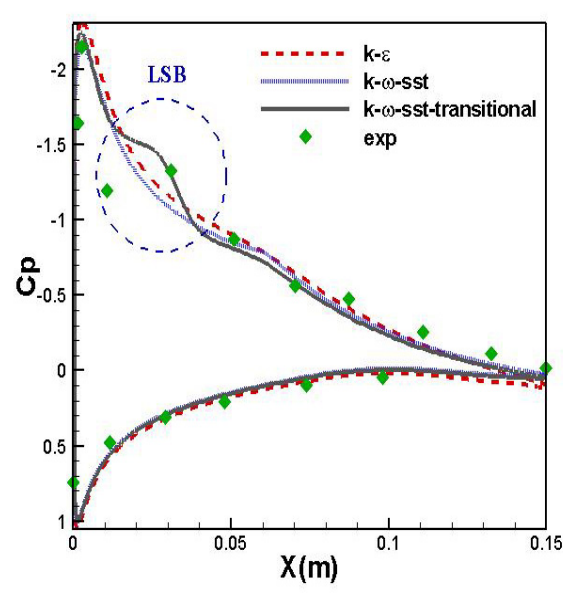

(a) $\alpha=8 \operatorname{deg}$

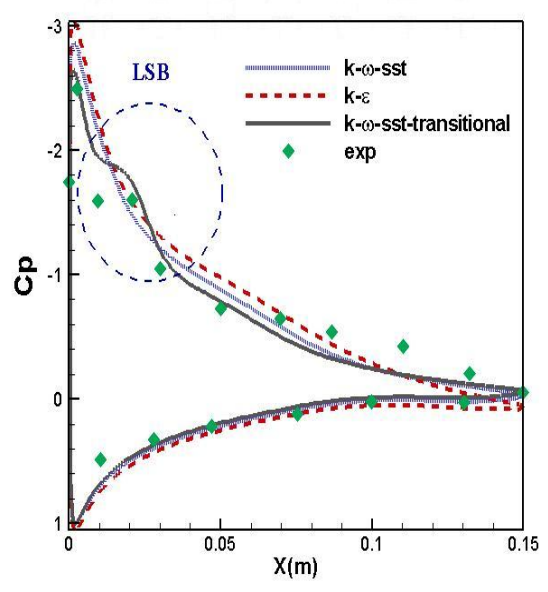

(b) $\alpha=10$ deg

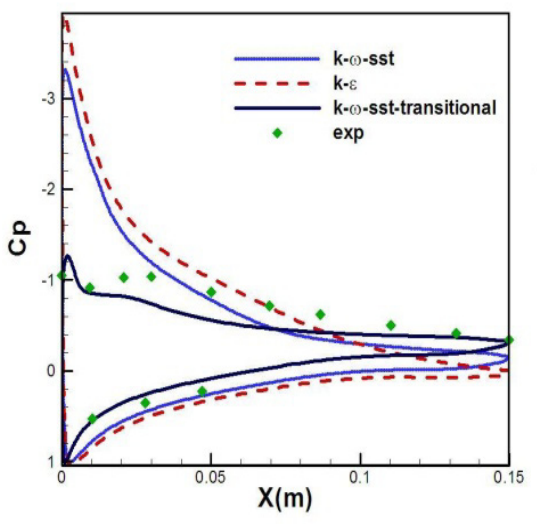

(c) $\alpha=12$ deg

Figure 2: $\quad$ Surface pressure distributions.

gradient amplified the disturbance in the separation zone and prompted transition and the bubble was shrinking in size. According to this model, at angle of attack of $10 \mathrm{deg}$, the separation position is at around $6 \%$ chord position and transition occurred at $14.2 \%$ of chord position. It is noticeable to say that, at this angle of attack the airfoil was close to stall therefore a large portion of the flow on the aerofoil was separated and the separated vortex formed near the trailing edge. Because of better prediction of $k-\omega$ SST transitional method, this model applied for the dynamic cases. 

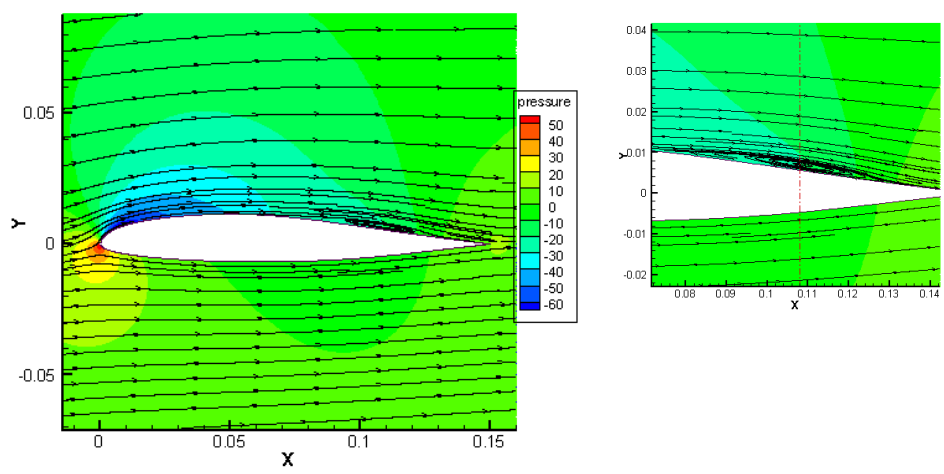

(a)
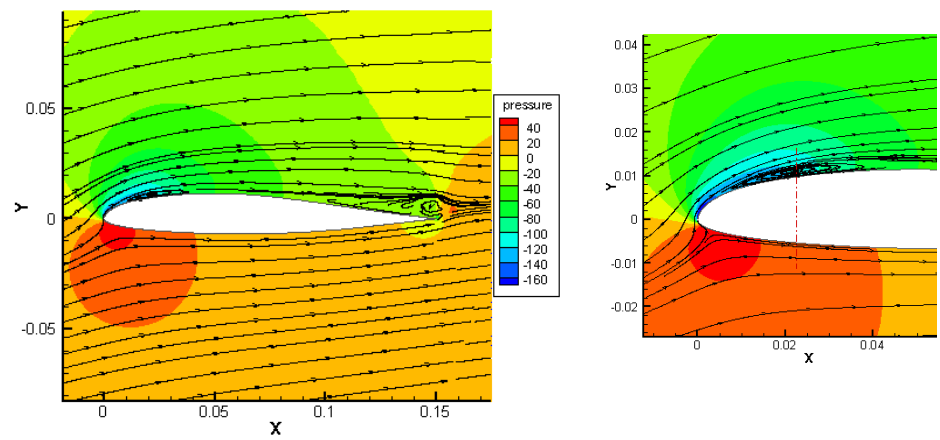

(b)

Figure 3: $\quad$ Static pressure and streamlines of $k-\omega$ SST transitional, $\alpha=4$ and 10 deg.

\subsection{Dynamic airfoil}

Numerical unsteady investigations of dynamic stall phenomenon were conducted on a plunging airfoil at mean angle of attack $12 \mathrm{deg}$ with amplitude of $8 \mathrm{~cm}$. The reduced frequency is set $\mathrm{k}=0.141$ and chord Reynolds number according to the static surveys is $10^{5}$. Figure 4 illustrates the sinusoidal plunging airfoil displacements of the experimental tests which were transformed in to equivalent angle of attack using the potential flow transformation formula $\bar{\alpha}_{e q}=i k \bar{h}$ also the mean angle of attack would be added to the equivalent angle. As it is clear in figure 4 , induced angle of attack has 90 degrees phase difference with vertical motion of the airfoil. It is noticeable to say that, the down stroke plunging displacement is chosen as the positive direction; besides there are four steps for the change of " $h$ " and corresponding " $\alpha_{\text {eq }}$ " such as (i) downward displacement of " $h$ " from $0-8 \mathrm{~cm}$ that $\alpha_{\max }<\alpha_{\mathrm{eq}}<0$, (ii) upward displacement of " $h$ " from $8 \mathrm{~cm}-0$, 
correspond to $0<\alpha_{\mathrm{eq}}<-\alpha_{\max }$, (iii) upward displacement of "h" from $0-(-8 \mathrm{~cm})$ correspond to $-\alpha_{\max }<\alpha_{\mathrm{eq}}<0$, (iv) downward displacement of "h" from (-8cm)-0, conformable to $0<\alpha_{\mathrm{eq}}<\alpha_{\max }$ thus, according to these sequences first half of the plunging oscillation cycle is related to the decreasing equivalent angle and the second one attributed to increasing equivalent angle. As pictured (figure 4) $\alpha_{\mathrm{eq}}$ is a maximum or minimum whenever $\mathrm{h}=0$ during down-stroke and upstroke motions respectively. In order to better understanding of the flow field all the results are presented according to the equivalent angle.

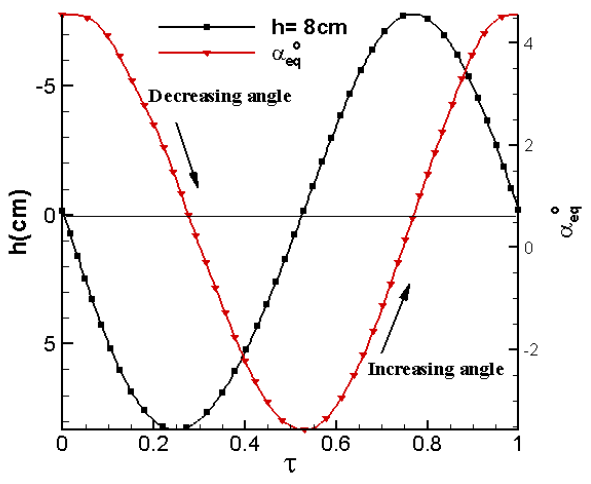

Figure 4: Time history of plunging motion with corresponding equivalent angle of attack.

Figure 5 (a-i) presents a chronology of the static fields superimposed on the instantaneous streamlines in order to depict the complicated vortex structures during the stall process. In the early stage of the increasing angle, the flow remained attached except in the trailing edge region, at $\alpha_{\mathrm{eq}} \approx 11.76^{\circ}$, Laminar Separation Bubble (LSB) close to the leading edge at $\mathrm{x} / \mathrm{c}=1.37$ was detected, as $\alpha_{\mathrm{eq}}$ increases beyond the $\alpha_{\mathrm{ss}}$ a thin flow reversal was observed in front of the trailing edge to leading edge, as one can observe from figures $5 \mathrm{~b}$ and $\mathrm{c}$ upstream of the flow reversal the boundary layer remained attached on the upper surface of the airfoil and the position of the LSB was moved toward the leading edge (for example, figure $5 \mathrm{~b}$ the center of the LBS changed to $\mathrm{x} / \mathrm{c}=0.11$ ) accordingly figure $5 \mathrm{~d}\left(\alpha_{\mathrm{eq}}=17.55 \mathrm{deg}\right)$ the turbulent boundary layer broke down and moved upstream and downstream, spillage the LSB, and initiated the formation of Leading edge vortex (LEV) afterward the LEV grew and covered rapidly downstream and covered the whole suction surface. During the further convection of the LEV due to the recirculation of this vortex, figure $5 \mathrm{~g}$-i, two secondary vortices near the leading edge were observed; aside from the vortices, a strong counter clockwise vortex appeared at the trailing edge toward the upper surface due to the low pressure region on the upper surface and high pressure difference on the trailing edge of lower surface which they pushed the flow from the lower surface and caused the CCW vortex; besides as $\alpha_{\mathrm{eq}}$ increased the trailing edge vortex was driven upward. 
62 Computational Methods and Experimental Measurements XV
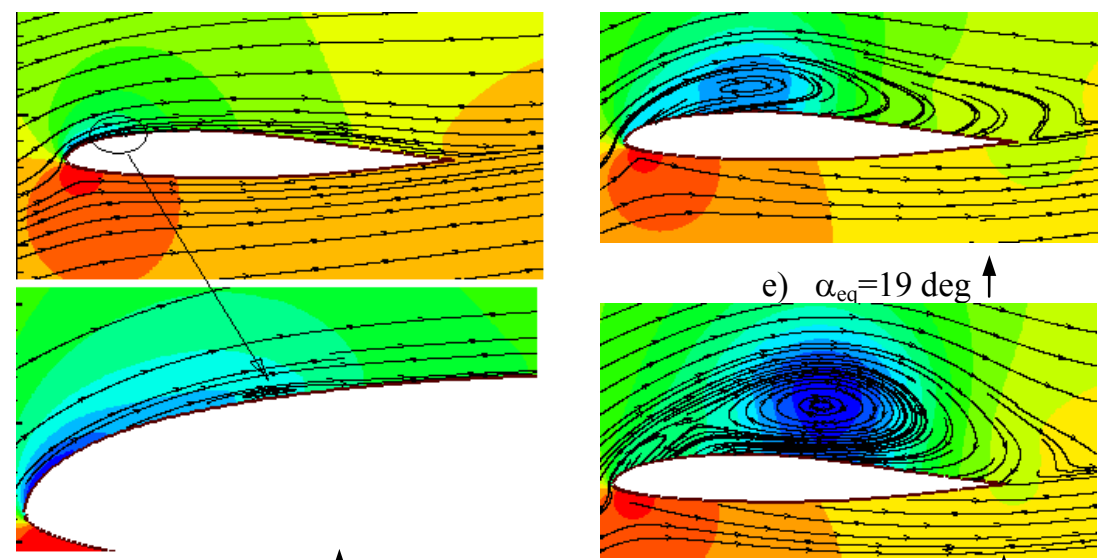

e) $\alpha_{\mathrm{eq}}=19 \mathrm{deg} \uparrow$

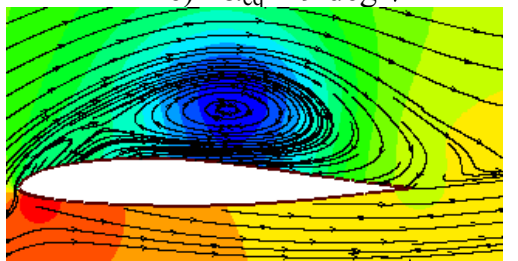

a) $\alpha_{\mathrm{eq}}=11.76 \mathrm{deg} \uparrow$

f) $\alpha_{\mathrm{eq}}=20.17 \mathrm{deg} \uparrow$
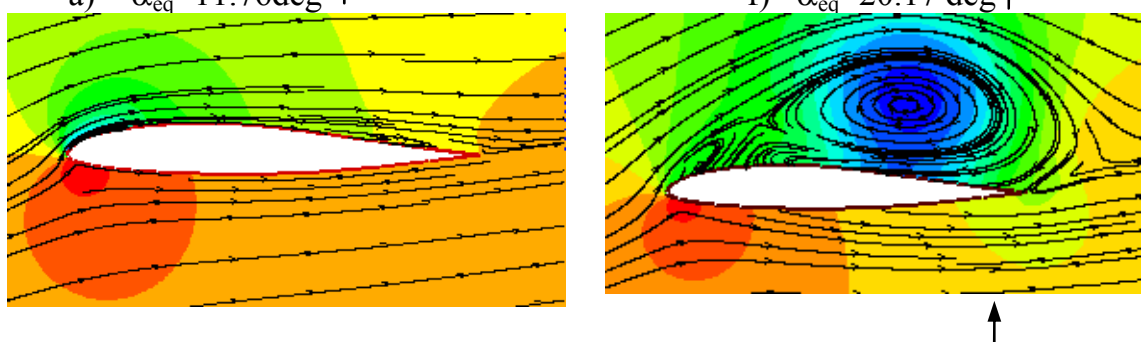

b) $\alpha_{\mathrm{eq}}=12.26 \mathrm{deg}$
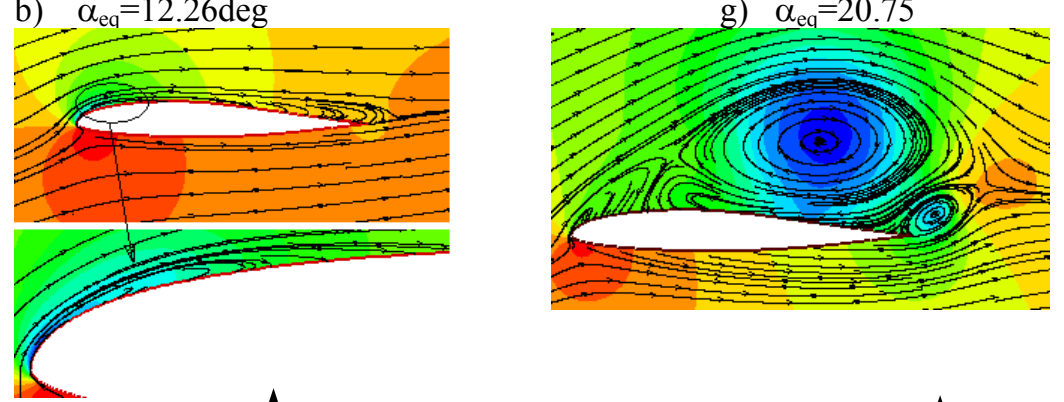

c) $\alpha_{\mathrm{eq}}=14.3 \mathrm{deg} \uparrow$

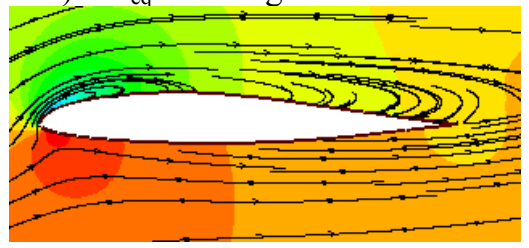

d) $\alpha_{\text {eq }}=17.55 \mathrm{deg} \uparrow$

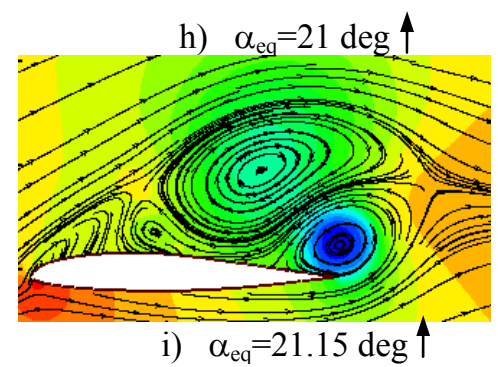

Figure 5: Pressure field superimposed on the instantaneous streamlines of increasing angle. 

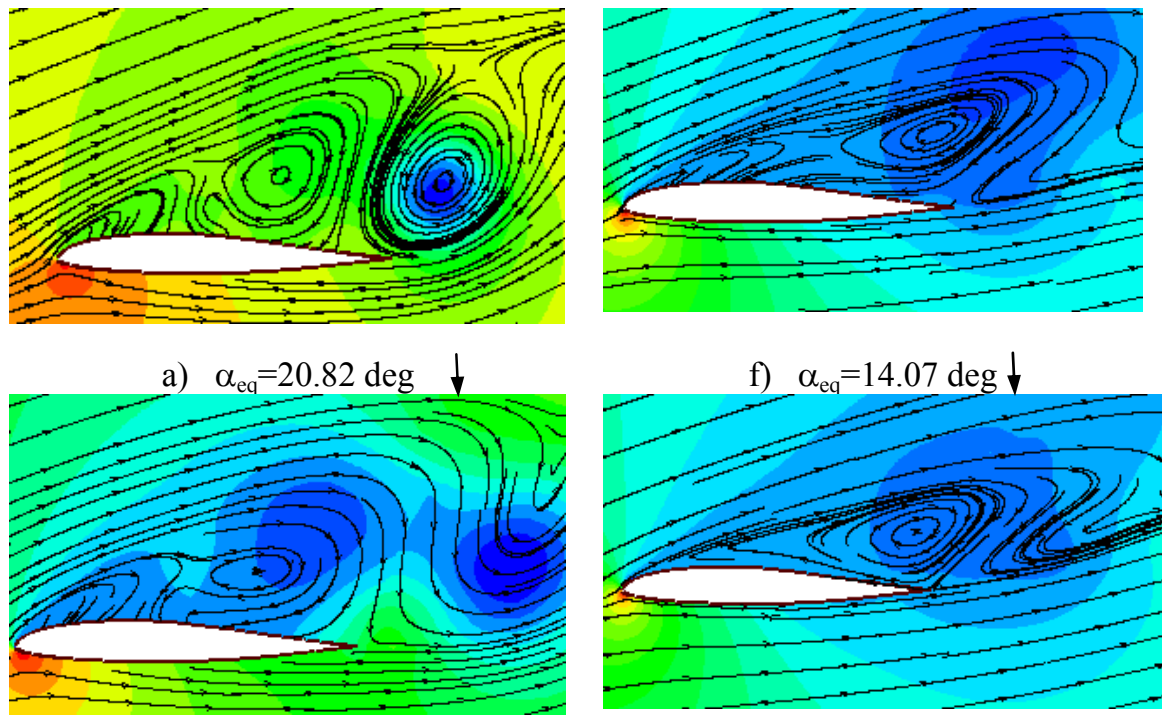

b) $\alpha_{\mathrm{eq}}=20.06 \mathrm{deg} \downarrow$

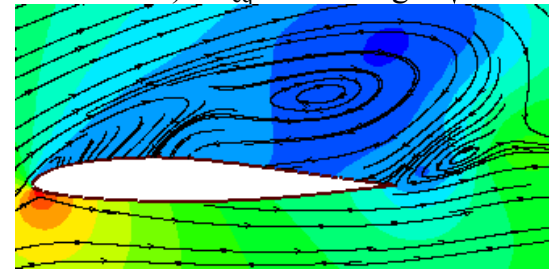

c) $\alpha_{\text {eq }}=19.2 \mathrm{deg}$

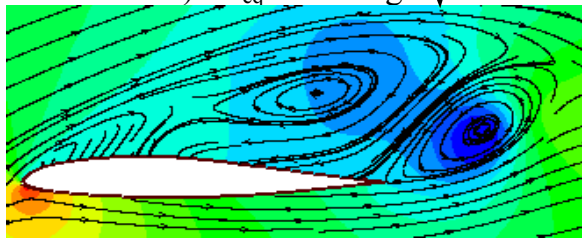

g) $\alpha_{\mathrm{eq}}=12.02 \mathrm{deg} \downarrow$

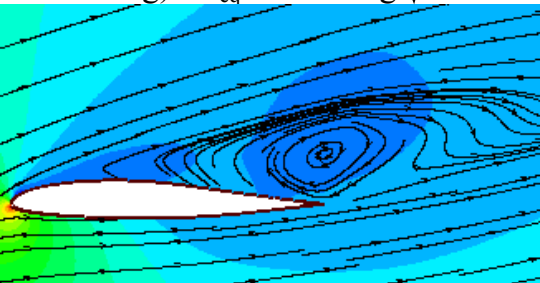

h) $\widehat{\alpha_{\mathrm{eg}}}=10 \mathrm{deg} \bar{\downarrow}$

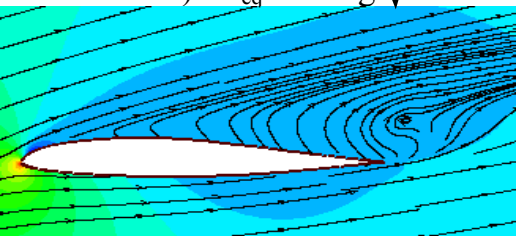

d) $\alpha_{e q}=18.15 \mathrm{deg}$

i) $\alpha_{\mathrm{eq}}=8.1 \mathrm{deg}$

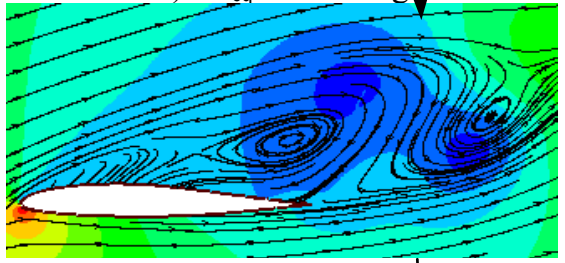

e) $\alpha_{\text {eq }}=15.54 \mathrm{deg}$

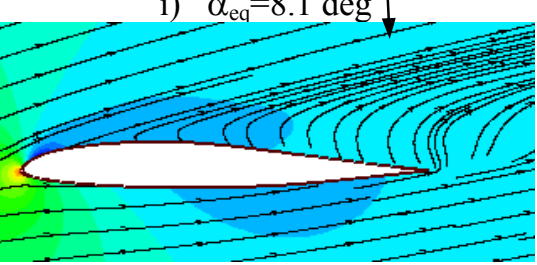

j) $\alpha_{\mathrm{eq}}=6.27 \mathrm{deg} \downarrow$

Figure 6: Pressure field superimposed on the instantaneous streamlines of decreasing angle. 
Figure 6a-j shows the static fields and instantaneous streamlines during decreasing angle. According to figure $6 \mathrm{a}\left(\alpha_{\mathrm{eq}}=20.82 \mathrm{deg}\right)$ in decreasing cycle, trailing edge vortex was grown and LEV became weaker, when $\alpha_{\mathrm{eq}}$ decreased further the trailing edge vortex disappeared and shed in to the flow (figure $6 \mathrm{~b}$ ) then the clockwise main vortex (growth LEV) moved away from the surface and again induced trailing edge vortex (figure $6 \mathrm{c}, \mathrm{d}$ and e) next the separated boundary layer flow reattached to the airfoil at lower incidences. It is significant to note that LEV shedding was forced by the motion in decreasing angle due to the inadequate time for fully develop of LEV before deceasing angle that had good agreement with experimental results [9].

Figure 7 compares numerical and experimental data at $\alpha_{\text {mean }}=12 \mathrm{deg}$ and $\mathrm{k}=0.141$, the hysteresis observed in the dynamic-pressure loops originated from the asymmetry in the locations or angles of attack, of the separation and there attachment. By inspecting the direction of the $\mathrm{C}_{\mathrm{p}}$ hysteresis loops, it is seen that the direction of the $\mathrm{C}_{\mathrm{p}}$ loops for both numerical and experimental data were clockwise, which means that the flow had lead phase. The pressure levels of the both results near the leading edge (figure $7 \mathrm{a}, \mathrm{b}$ ) were almost the same in increasing cycle and the $\mathrm{Cp}$ slopes were nearly identical but in decreasing stroke the results were deviated from the experimental data.This is mainly because of complicated separated flow and vortex shedding structure. Note that apart from the small divergence, these two graphs rendered same pattern in decreasing angle which indicted, this model could well predict boundary layer events, LEV, separation, detaching and reattachment, in addition the Transition SST model presents a too sharp drop-off of the $\mathrm{C}_{\mathrm{p}}$ when the dynamic stall occurs giving an over-prediction of the strength of the stall. Figure $7 \mathrm{c}, \mathrm{d}$ depicts the pressure distributions of the ports $\mathrm{x} / \mathrm{c}=20 \%$ and $50 \%$, according to this data narrower hysteresis (compared to the leading edge port) was recorded because of massive separation of the flow on the upper surface of the airfoil. In the aft portion of the airfoil figure $8 \mathrm{~d}$, the experimental pressure loops were similar to "figure 8 shape" moreover $\left|C_{P \max }\right|$ of numerical method at this position was recorded 2.9 while it was 1.63 for the experimental case which means the numerical method predicted less dissipative LEV in process.

Figure 8 shows the numerical dynamic stall process of $C_{1}$ versus $\alpha_{e q}$ at $\alpha_{\text {mean }}=12 \mathrm{deg}$ and $\mathrm{k}=0.141$, the validity of these processes were published by McCroskey [3], McCroskey et al. [2], McAlister et al. [10], Lee and Gerontakos [11-12]. According to Lee and Gerontakos nomenclature point 1and 2 corresponded to the initiation and ending of the upward spread of the flow reversal respectively besides between the points 2 an 3 turbulence bread down, initiation and growth of the LEV were occurred and the lift coefficient underwent a sharp drop as soon as the LEV passed off the trailing edge, and the airfoil remained fully stalled point 3 and 4 . At point 5 (decreasing $\alpha_{\mathrm{eq}}=20.1$ ) there was a slight increase in $\mathrm{C}_{1}$ owing to the presence and convection of the secondary vortex. According to explanations k- $\omega$-SST transitional method could predict the boundary layer event with reasonable accuracy. 


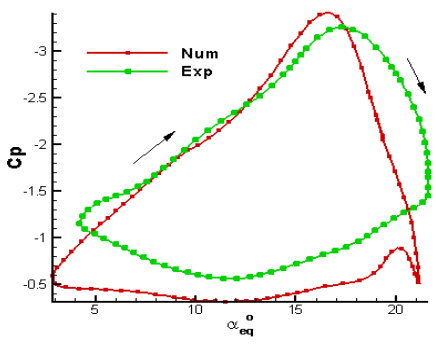

a) $5 \%$

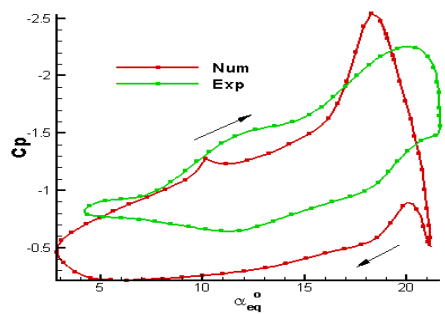

c) $20 \%$

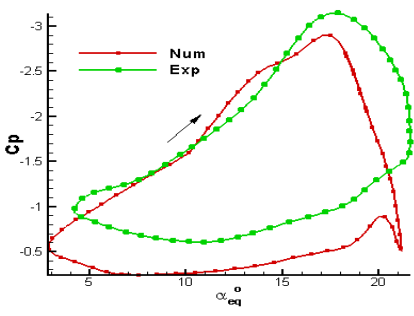

b) $10 \%$

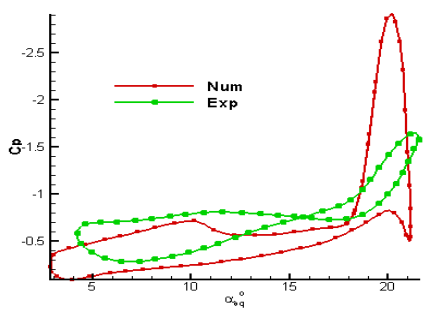

d) $50 \%$

Figure 7: Comparison of numerical and experimental pressure coefficient hysteresis loops.

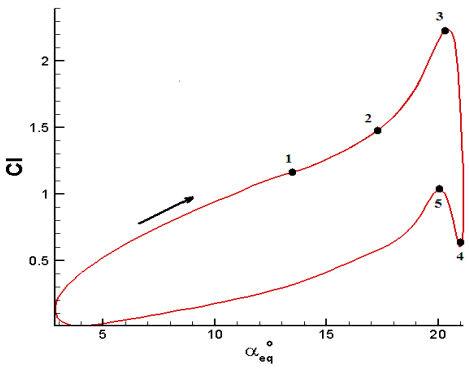

Figure 8: Variations of numerical dynamic lift coefficient.

\section{Conclusions}

In this paper static and dynamic flow field on an Eppler 361 airfoil at $\mathrm{Re}=10^{5}$ were investigated. For a static airfoil different turbulence methods analyzed 
consequently, k- $\omega$ SST transitional model was capable of predicting the flow characteristics such as LSB and trailing edge separation better than other models therefore, in general this model employed to simulate the fluid flow around an E361 airfoil executing a sinusoidal plunging motion. In the sequence of dynamic motion LSB, reverse flow, turbulence breakdown, spillage of LEV, secondary vortices and detachment of the fluid were captured accurately while the dissipation of the progressive LEV was less than experimental data. In the increasing cycle pressure levels of the both results near the leading edge were almost the same however in decreasing stroke the numerical data deviated from the experimental counterparts.

\section{References}

[1] P. Wernert, W. Geissler, M. Raffel, and J. Kompenhans, Experimental and numerical investigations of dynamic stall on a pitching airfoil, AIAA journal, vol. 34, pp. 982-989, 1996.

[2] McCroskey, W. J., McAlister, K. W., Carr, L. W., Pucci, S. L., Lamber, O., and Indergrand, R. F., Dynamic Stall on Advanced Airfoil Sections, Journal of American Helicopter Society, Vol. 26, July 1981, pp. 40-50.

[3] McCroskey, W. J., Unsteady Airfoils, Annual Review of Fluid Mechanics, Vol. 14, 1982, pp. 285-311.

[4] Ericsson, L. E., and Reding, J. P., Fluid Mechanics of Dynamic Stall. Part I. Unsteady Flow Concepts, Journal of Fluids and Structures, Vol. 2,Jan. 1988, pp. 1-33.

[5] Lee, T., and Basu, S., Measurement of Unsteady Boundary Layer Developed on an Oscillating Airfoil Using Multiple Hot-Film Sensors, Experiments in Fluids, Vol. 25, No. 2, 1998, pp. 108-117.

[6] S. Wang, L. Ma, D. Ingham, M. Pourkashanian, and Z. Tao, Numerical Investigations on Dynamic Stall Associated with Low Reynolds Number Flows over Airfoils, in The 2010 International Conference On Mechanical and Aerospace Engineering (CMAE 2010) Chengdu, China, 2010.

[7] Ajalli F., Mani M., Soltani M. An Experimental Investigation of Pressure Distribution around a Heaving Airfoil, The 5th International conference on Heat Transfer, Fluid Mechanics and Thermodynamics, South Africa, Spring 2007.

[8] Fluent 6.3,26 (Theory Guide),"

[9] M. Mani, F. Ajalli M. R. Soltani, An experimental investigation of the reduced frequency effects into pressure coefficients of a plunging airfoil, Advances in Fluid Mechanic 2008.

[10] McAlister, K. W., Carr, L. W. \& McCroskey, W. J., Dynamic stall experiments on the NACA 0012 airfoil, NASA TP 1100. 1978.

[11] Lee, T., and Gerontakos, P., Investigation of Flow over an Oscillating Airfoil, Journal of Fluid Mechanics, Vol. 512, 2004, pp. 313-341.

[12] T. Lee, P. Gerontakos, Investigation of flow over an oscillating airfoil, J. Fluid Mech. (2004), vol. 512, pp. 313-341 